\title{
Empagliflozin in heart failure patients with reduced ejection fraction: a randomized clinical trial (Empire HF)
}

Jesper Jensen ${ }^{1,7^{*}}$ (D), Massar Omar2,8, Caroline Kistorp ${ }^{3,7}$, Mikael Kjær Poulsen², Christian Tuxen ${ }^{4}$, Ida Gustafsson ${ }^{4,7}$, Lars Køber ${ }^{5,7}$, Finn Gustafsson ${ }^{5,7}$, Emil Fosbøl ${ }^{5}$, Niels Eske Bruun ${ }^{6,7,9}$, Lars Videbæk ${ }^{2}$, Peter Hartmund Frederiksen², Jacob Eifer Møller ${ }^{2,8}$ and Morten Schou ${ }^{1,7}$

\begin{abstract}
Background: Data from recent cardiovascular outcome trials in patients with type 2 diabetes (T2D) suggest that sodium-glucose cotransporter 2 (SGLT2) inhibitors can prevent development of heart failure (HF) and prolong life in patients without HF. Ongoing event-driven trials are investigating whether the same effect is present in patients with well-defined HF. The mechanism behind the effect of SGLT2 inhibitors in patients with T2D and the potential effect in patients with overt HF is presently unknown.

Methods: This is a randomized, double-blinded, placebo-controlled, parallel group, clinical trial including HF patients with reduced left ventricular ejection fraction (HFrEF) with an ejection fraction $\leq 40 \%$ on optimal therapy recruited from specialized HF clinics in Denmark. The primary aim is to investigate the effect of the SGLT2 inhibitor empagliflozin on N-terminal pro-brain natriuretic peptide (NT-proBNP). Secondary endpoints include cardiac biomarkers, function and hemodynamics, metabolic and renal parameters, daily activity level, and quality of life. Patients are assigned 1:1 to 90 days treatment with empagliflozin $10 \mathrm{mg}$ daily or placebo. Patients with $\mathrm{T} 2 \mathrm{D}$ are required to be on recommended doses of anti-glycemic therapy with a hemoglobin A1c ( $\mathrm{HbA1c}$ ) of $6.5-10.0 \%(48-86 \mathrm{mmol} / \mathrm{mol})$. To show a betweengroup difference in the change of NT-proBNP of 30\%, a total of 189 patients will be included.
\end{abstract}

Discussion: The Empire HF trial will elucidate the effects and modes of action of empagliflozin in HFrEF patients with and without T2D and provide important mechanistic data which will complement ongoing event-driven trials.

Trial registration: Clinicaltrialsregister.eu, EudraCT Number 2017-001341-27. Registered on 29 May 2017. ClinicalTrials.gov, NCT03198585. Registered on 26 June 2017.

Keywords: Heart failure, SGLT2 inhibitors, Mechanism, Mode of action, NT-proBNP, Daily activity level, Cardiac function, Metabolic endpoints, Renal endpoints, Quality of life

\section{Background}

Within recent years, attention to heart failure (HF) care in patients with type 2 diabetes (T2D) has increased markedly after results from three randomized clinical trials (RCT) evaluating the effect of sodium-glucose co-transporter 2 (SGLT2) inhibitors [1-3]. In these safety trials, it was observed that three different SGLT2 inhibitors could prevent

\footnotetext{
*Correspondence: jesper.jensen.06@regionh.dk

'Department of Cardiology, Herlev-Gentofte Hospital, Herlev Ringvej 75, 2730 Herlev, DK, Denmark

${ }^{7}$ Faculty of Health and Medical Sciences, Copenhagen University, Blegdamsvej 3B, 2200 København N, DK, Denmark

Full list of author information is available at the end of the article
}

development of HF and prolong life in patients with T2D. Recently, the results have been replicated in real life $[4,5]$. The mechanism behind these observations are poorly understood and while several hypotheses have been proposed, data are lacking [6-9].

In the Empagliflozin, Cardiovascular Outcomes, and Mortality in Type 2 Diabetes (EMPA-REG OUTCOME) trial, the Canagliflozin Cardiovascular Assessment Study (CANVAS) program, and the Dapagliflozin Effect on Cardiovascular Events-Thrombolysis in Myocardial Infarction 58 (DECLARE-TIMI 58) trial, $10-14 \%$ of the included patients had a reported history of HF at baseline and a

(c) The Author(s). 2019 Open Access This article is distributed under the terms of the Creative Commons Attribution 4.0 International License (http://creativecommons.org/licenses/by/4.0/), which permits unrestricted use, distribution, and 
significant subgroup effect was observed with a reduction in the composite endpoint of hospitalization for $\mathrm{HF}$ or cardiovascular death in patients treated with empagliflozin, canagliflozin, and dapagliflozin, respectively $[3,10,11]$. However, HF was neither well-defined nor described in these trials; central information such as whether the patients with reported HF had preserved (HFpEF) or reduced (HFrEF) ejection fraction is unknown. The possible beneficial effects of SGLT2 inhibitors are unknown in patients without T2D but the primary action in the kidneys - induction of glucosuria and natriuresis - have been demonstrated in individuals without T2D [12]. Proposed derived mechanisms potentially explaining the observed cardiovascular benefits of SGLT2 inhibitors include a proportionally larger reduction in the extracellular volume (ECV) than in the plasma volume (PV) $[13,14]$, leading to a reduced preload, left ventricular (LV) filling pressure, and LV wall stress, the latter being the primary driver for $\mathrm{N}$-terminal pro-brain natriuretic peptide (NT-proBNP) production. Thereby, a net effect of decreased NT-proBNP levels, which is associated with a decreased mortality risk in HF populations, is plausible $[15,16]$ and a decrease of $30 \%$ in NT-proBNP has previously been shown to be clinically significant in this population [17]. Other proposed derived mechanisms of SGLT2 inhibitors supporting beneficial cardiovascular effects in HF patients include a favorable shift in glucose and fat metabolism towards increased ketone substrate use [18, 19], renoprotective effects with changes in intrarenal hemodynamics, uricosuria and reduced albuminuria [7], and direct cardiac effects with remodeling of the myocardium ultimately leading to an improvement in the ventricular systolic function [9].

To increase the understanding of the cardiovascular effects of SGLT2 inhibitors, we are evaluating the effect of the SGLT2 inhibitor empagliflozin in HFrEF patients with a left ventricular ejection fraction (LVEF) $\leq 40 \%$ on cardiac biomarkers, function and hemodynamics, metabolic and renal parameters, and daily activity level and quality of life. The results of the Empire HF trial will complement the results from ongoing event-driven trials and may provide pathophysiological insight into the effect of this new group of drugs in HFrEF patients. Here, we present the protocol for the Empire HF trial. A checklist in accordance with the Standard Protocol Items: Recommendations for Interventional Trials (SPIRIT) for the reporting of the protocol is available (Additional file 1).

\section{Methods}

\section{Study hypotheses}

The study hypotheses are presented in Fig. 1. The main hypothesis of the Empire HF trial is that 90 days treatment with empagliflozin $10 \mathrm{mg}$ once daily compared with placebo reduces NT-proBNP in stable HFrEF patients on optimal therapy. An exploratory hypothesis is that the treatment is associated with an increase in the daily activity level and secondary hypotheses include that the treatment: reduces the amount of visceral fat, reduces insulin resistance, and increases supply of ketones to the heart (Metabolic hypothesis); maintains glomerular filtration rate (GFR) and reduces estimated extracellular volume (eECV), estimated plasma volume (ePV), uric acid, and urinary excretion of albumin (Renal hypothesis); reduces plasma concentrations of midregion pro-peptide of adrenomedullin (MR-proADM) and high-sensitivity cardiac troponin I (hs-cTnI) (Cardiac Biomarker hypothesis); improves left ventricular global longitudinal strain (LV-GLS) and LVEF at rest and during pharmacological stress (Cardiac function hypothesis); reduces the pulmonary capillary wedge pressure (PCWP) to cardiac index (CI) ratio during sub-maximal exercise and improves LV contractile reserve (Hemodynamic hypothesis); improves healthrelated quality of life (Quality of life hypothesis).

\section{Primary objective}

The primary objective of the study is to assess the effect of empagliflozin on NT-proBNP.

\section{Secondary objectives}

An exploratory objective of the study is to assess the effect of empagliflozin on daily activity level. Secondary objectives include assessment of the effects on body composition, glucose metabolism, and ketones; GFR, eECV, ePV, urid acid, and urine albumin to creatinine ratio (UACR); cardiac biomarkers including MR-proADM and hs-cTnI; LV diastolic and systolic function at rest and during lowdose dobutamine infusion with echocardiography; central invasive hemodynamics at rest and during exercise; and health-related quality of life.

\section{Study design}

Investigator-initiated, double-blinded, placebo-controlled, parallel group RCT. Two experimental sites and five recruiting sites.

\section{Study population}

Stable outpatients with HFrEF on optimal therapy in accordance with most recent European and national guidelines [20]. It is expected that $20 \%$ of the patients have known T2D and that an additional $30 \%$ of the patients will have a diagnosis of new onset T2D or impaired glucose tolerance based on an oral glucose tolerance test (OGTT) at the randomization visit [21, 22].

\section{Primary endpoint}

Between-group difference in the change of plasma concentrations of NT-proBNP from baseline to 90 days. 


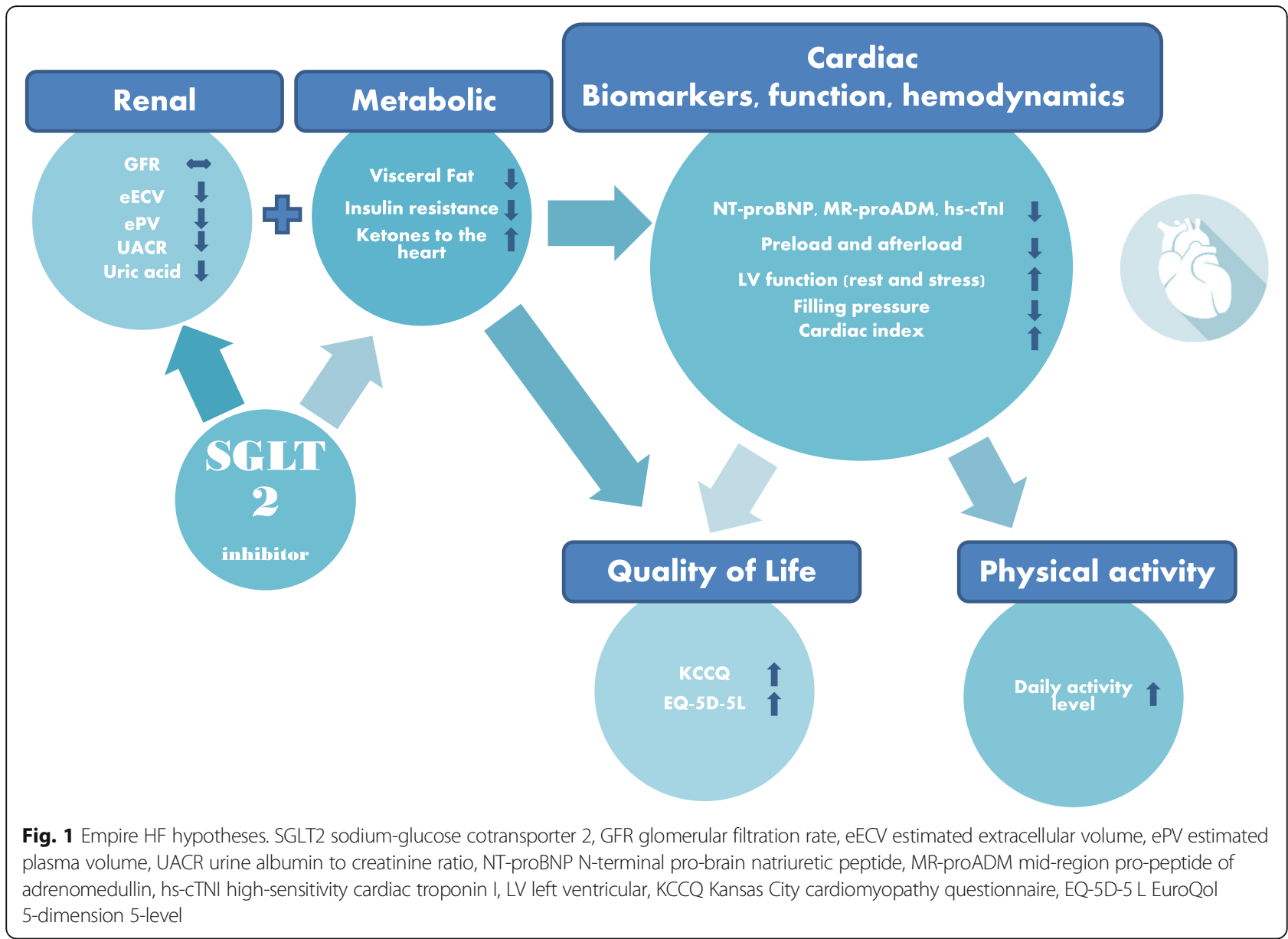

\section{Secondary endpoints}

All the below secondary endpoints will be quantified as the between-group difference in the change of the given endpoint from baseline to 90 days. As an exploratory endpoint, the amount of daily average accelerometer units is chosen, measured by patient-worn accelerometers [23]. Secondary endpoints include: visceral fat assessed by whole-body dual-energy X-ray absorptiometry (DXA) scan [24]; ePV assessed by hematocrit and hemoglobin [25]; glucose metabolism assessed by OGTT including assessment of insulin sensitivity quantified with the Matsuda index [21]; ketone supply to the heart assessed by measuring betahydroxybutyrate [26, 27]; renal function assessed by measuring GFR and eECV with chromium-51 labelled ethylenediamine tetraacetic acid $\left({ }^{51} \mathrm{Cr}\right.$-EDTA) clearance [28, 29]; uric acid and UACR [30, 31]; cardiac biomarkers assessed by measuring MR-proADM and hs-cTnI [32, 33]; cardiac systolic and diastolic function including LV-GLS and LVEF assessed by transthoracic echocardiography at rest and during low-dose dobutamine stress with a dosage of $20 \mu \mathrm{g} / \mathrm{kg} /$ min [34, 35]; cardiac hemodynamics during sub-maximal exercise assessed by right heart catheterization (RHC) including PCWP/CI index and LV contractile reserve [36]; and health-related quality of life assessed by the questionnaires Kansas City Cardiomyopathy Questionnaire (KCCQ) and EuroQol 5-dimension 5-level (EQ-5D-5 L) questionnaire $[37,38]$.

\section{Assessment and randomization}

The schedule of enrolment, interventions, and assessments in accordance with SPIRIT is outlined in Fig. 2. At the screening visit, informed consent is obtained by an investigator or trained study nurse, baseline characteristics assessed based on the medical record and confirmed in consultation with the patient, and eligibility confirmed with fulfilment of all inclusion criteria and no exclusion criteria. At the following randomization visit, patients are randomized 1:1 regarding both the primary and secondary endpoints. To ensure both safety assessment, adherence, and complete follow-up, follow-up assessments are carried out as two telephone contacts, a control visit, and an end-of-study visit. The allocated treatment of individual patients may be discontinued or modified based on the patient's decision, a positive pregnancy test, severe non-adherence, or if the investigators assess that further treatment is contraindicated because 


\begin{tabular}{|c|c|c|c|c|c|c|}
\hline & $\begin{array}{c}\text { Visit } 1 \\
\text { Screening }\end{array}$ & $\begin{array}{c}\text { Visit } 2 \\
\text { Randomization }\end{array}$ & $\begin{array}{l}\text { Phone } \\
\text { contact }\end{array}$ & $\begin{array}{c}\text { Visit } 3 \\
\text { Clinical control }\end{array}$ & $\begin{array}{l}\text { Phone } \\
\text { contact }\end{array}$ & $\begin{array}{c}\text { Visit } 4 \\
\text { Study closure }\end{array}$ \\
\hline Time (days) & $-30 /-3$ & 0 & $20 \pm 5$ & $45 \pm 10$ & $70 \pm 5$ & $90 \pm 15$ \\
\hline \multicolumn{7}{|l|}{ General } \\
\hline Informed consent form & $x$ & & & & & \\
\hline Inclusion and exclusion criteria & $\mathrm{X}$ & $\mathrm{x}$ & & & & \\
\hline Demography & $x$ & & & & & \\
\hline Medical and surgical history & $x$ & & & & & \\
\hline General physical examination & $x$ & & & & & $\mathrm{x}$ \\
\hline Targeted physical examination & & $x$ & & $x$ & & \\
\hline Vital signs & $\mathrm{x}$ & $\mathrm{x}$ & & $\mathrm{x}$ & & $\mathrm{x}$ \\
\hline 12-ECG & $\mathrm{x}$ & & & & & \\
\hline Pregnancy test (if relevant) & $\mathrm{X}$ & $\mathrm{X}$ & & & & $\mathrm{X}$ \\
\hline \multicolumn{7}{|l|}{ Endpoints } \\
\hline Blood samples (fasting) & & $x$ & & & & $x$ \\
\hline Urine sample (morning) & & $x$ & & & & $x$ \\
\hline Transthoracic echocardiography & & $\mathrm{x}$ & & & & $\mathrm{x}$ \\
\hline OGTT & & $x$ & & & & $x$ \\
\hline Accelerometry & & $\mathrm{X}(-30$ days $)$ & & & & $\mathrm{x}$ \\
\hline DXA-scan ${ }^{\#}$ & & $\mathrm{x}$ & & & & $x$ \\
\hline${ }^{51} \mathrm{Cr}$-EDTA clearance ${ }^{\#}$ & & $\mathrm{x}$ & & & & $\mathrm{x}$ \\
\hline Dobutamine stress echocardiography & & $\mathrm{x}$ & & & & $\mathrm{x}$ \\
\hline Right heart catheterization ${ }^{*}$ & & $X( \pm 15$ days $)$ & & & & $x$ \\
\hline \multicolumn{7}{|l|}{ Safety } \\
\hline AEs and SAEs & & & $\mathrm{x}$ & $\mathrm{x}$ & $\mathrm{x}$ & $x$ \\
\hline Blood samples (non-fasting) & $\mathrm{x}$ & & & $\mathrm{x}$ & & \\
\hline \multicolumn{7}{|l|}{ Medication } \\
\hline Concomitant (adherence) & $\mathrm{x}$ & $\mathrm{x}$ & $\mathrm{x}$ & $\mathrm{x}$ & $\mathrm{x}$ & $\mathrm{x}$ \\
\hline IP (adherence) & & & $x$ & $x$ & $x$ & $x$ \\
\hline
\end{tabular}

Fig. 2 Schedule of enrolment, interventions, and assessments in accordance with the Standard Protocol Items: Recommendations for Interventional Trials (SPIRIT). "Only performed in the patients enrolled at Herlev-Gentofte Hospital. "Only performed in a sub-group of the patients enrolled at Odense University Hospital. ECG electrocardiogram, OGTT oral glucose tolerance test, DXA dual-energy X-ray absorptiometry, ${ }^{51} \mathrm{Cr}$ EDTA chromium-51 labeled ethylenediamine tetra-acetic acid. AE adverse event, SAE severe adverse event, IP investigational product

of adverse events (AEs) or other safety reasons. If possible, patients who discontinue or deviate from the protocol will be followed up regarding all endpoints. Strategies to improve adherence include information at both the screening and randomization visit regarding correct drug administration and the patients are encouraged to use a medication diary or pill boxes during the study. At all planned contacts during the treatment period, the patients are asked whether they adhere to the correct drug administration. At the end of the study visit, excess tablets of the investigational product (IP) are returned for drug accountability.

\section{Inclusion and exclusion criteria}

Inclusion and exclusion criteria are presented in Table 1. Both HF patients with and without T2D are included. Patients with known T2D will be treated in accordance with European and national guidelines and will be on recommended, stable dose(s) of anti-glycemic drug(s) for 30 days before randomization and no additional anti-glycemic drugs will be added during the study period. Patients with new onset T2D at the randomization visit will not receive additional anti-glycemic treatment during the study period. Regarding treatment for HF, patients will be on optimal, stable medical treatment in accordance with European guidelines for 30 days before randomization. If indicated, a device will be implanted before randomization in accordance with national guidelines. For implantation of a cardioverter defibrillator (ICD) and cardiac resynchronization therapy (CRT), a minimum period of 30 and 90 days will be obtained between implantation and randomization, respectively. During the study period, conventional HF therapy will only be changed if side effects are suspected.

\section{Schedule of enrolment, interventions, and assessments}

Eligible patients will undergo the study visits presented in Fig. 2. In addition, unlimited access to telephone and email service is available and, if considered necessary, unscheduled visits during the study period through four weeks after the end-of-study visit is planned. Follow-up will be performed by investigators, sub-investigators, and study nurses educated in the specialized treatment of HF patients and who are trained in the study protocol. Important protocol 
Table 1 Inclusion and exclusion criteria

\begin{tabular}{|c|c|}
\hline Inclusion criteria & Exclusion criteria \\
\hline $\begin{array}{l}\text { Optimal heart failure therapy in } \\
\text { accordance with European and } \\
\text { national guidelines }\end{array}$ & CRT-D/-P implanted $<90$ days \\
\hline LVEF $\leq 0.40$ & $\begin{array}{l}\text { Uncorrected severe valvular } \\
\text { disease }\end{array}$ \\
\hline $\mathrm{eGFR}>30 \mathrm{~mL} / \mathrm{min} / 1.73 \mathrm{~m}^{2}$ & Non-compliance \\
\hline $\mathrm{BMl}<45 \mathrm{~kg} / \mathrm{m}^{2}$ & Use of metalozone \\
\hline NYHA class I-III & NYHA class IV \\
\hline \multirow[t]{3}{*}{ Age $>18$ years } & Age $>85$ years \\
\hline & Dementia \\
\hline & Admission for $\mathrm{HF}<30$ days \\
\hline $\begin{array}{l}\text { If T2D - optimal treatment in } \\
\text { accordance with European } \\
\text { and national guidelines }\end{array}$ & $\begin{array}{l}\text { Admission for hypoglycemia } \\
<12 \text { months }\end{array}$ \\
\hline $\begin{array}{l}\text { If } \mathrm{T} 2 \mathrm{D} \text { - stable doses of } \\
\text { anti-glycemic treatment } \\
\text { for } 30 \text { days }\end{array}$ & Known sustained $\mathrm{VT}$ \\
\hline \multirow[t]{6}{*}{ If T2D - HbA1c 6.5-10.0\% } & $\begin{array}{l}\text { Symptomatic hypotension } \\
\text { and systolic BP }<95 \mathrm{mmHg}\end{array}$ \\
\hline & $\begin{array}{l}\text { Unable to perform an exercise } \\
\text { test }\end{array}$ \\
\hline & Immobilization \\
\hline & Pregnancy \\
\hline & $\begin{array}{l}\text { Participation in other medical } \\
\text { trials }\end{array}$ \\
\hline & $\begin{array}{l}\text { Previous intolerance of } \\
\text { Empagliflozin or excipients }\end{array}$ \\
\hline
\end{tabular}

LVEF left ventricular ejection fraction, eGFR estimated glomerular filtration rate, $B M I$ body mass index, NYHA New York Heart Association, T2D type 2 diabetes, $H b A 1 c$ hemoglobin $A 1 C, C R T-D /-P$ cardiac resynchronization therapy with defibrillator (-D) or without defibrillator (-P), $H F$ heart failure, $V T$ ventricular tachycardia, $B P$ blood pressure

modifications will be communicated to relevant parties including regulators, the ethical review board, investigators, trial participants, trial registries, and journals.

\section{Statistical analyses}

Intention-to-treat (ITT) analyses will be applied as the primary analysis. Analysis of the primary endpoint is comparison of the between-group difference in the change of NT-proBNP from baseline to 90 days. The primary endpoint will be analyzed using analysis of covariance (ANCOVA), with treatment as a fixed factor, the baseline NT-proBNP level as a covariate and with adjustment for age, sex, history of T2D, and site of randomization. Missing data in the primary analysis will be estimated using imputation. Approximately half the patients will have either known T2D, new-onset T2D, or impaired glucose tolerance. Test for interaction between treatment, abnormal glucose tolerance, and all the specified endpoints (predefined sub-group analyses) will be performed. Log transformation will be performed if assumption of normality is not met. After log transformation, the parameter will be further tested for normality as indicated. A two-tailed $p$ value $\leq 0.05$ is considered statistically significant. Normally distributed variables will be presented as mean \pm standard deviation (SD) and skewed distributed variables as median and interquartile range [IQR]. Comparisons between treatment and placebo group will be performed by an unpaired two sample $t$-test, Mann-Whitney test, or $x^{2}$ test as appropriate.

\section{Sample size}

Primary endpoint: Based on data from a previous study, it is expected that a reduction in NT-proBNP of $30 \%$ is clinically significant [17]. To test the primary hypothesis that empagliflozin $10 \mathrm{mg}$ daily compared with placebo reduces NT-proBNP with $30 \%$ (SD of 70\%) with a power of 0.80 and a significance level of 0.05 , a total of $N=172$ patients in the main study is required. To allow for dropouts, the final total sample size is planned to be 189 patients.

Low dose dobutamine sub-study: To detect a clinically significant decrease in LV-GLS of $20 \%$ (SD of 30\%) in patients treated with empagliflozin compared with placebo in this secondary endpoint with a power of 0.80 and a significance level of 0.05 , a total of 72 patients are required. To allow for dropouts, $N=119$ patients are planned to be enrolled at Herlev-Gentofte Hospital in this sub-study (Cardiac function hypothesis).

Hemodynamic sub-study: PCWP is expected to increase to $32 \pm 8 \mathrm{mmHg}$ and $\mathrm{CI}$ to $5.0 \pm 1.4 \mathrm{~L} / \mathrm{min} / \mathrm{m}^{2}$ with exercise, with a PCWP/CI ratio equal to $6.4 \pm 1.4$. To detect a decrease of $20 \%$ in patients treated with empagliflozin compared with placebo in this secondary endpoint with a power of 0.80 and a significance level of 0.05 , a total of 61 patients are required. To allow for dropouts, $N=70$ patients are planned to be enrolled at Odense University Hospital in this sub-study (Hemodynamic hypothesis).

In all the other sub-studies, the sample size is a consequence of the main study on NT-proBNP and confidence intervals will be evaluated critically. Based on previous studies it is expected to observe a possible significant difference with the used sample sizes [24, 39].

\section{Blinding}

Both patients and investigators are blinded to the treatment allocation. The IP of empagliflozin $10 \mathrm{mg}$ tablets or matching placebo are produced by the Glostrup Pharmacy as identical white capsules delivered to each patient as a container with 90 capsules. The Glostrup Pharmacy is a public pharmacy which is independent of the steering committee and study centres. The IP is produced and controlled in accordance with the requirements in the Commission Directive 2003/94/EC of 8 October 2003 laying down the principles and guidelines of good manufacturing practice and in compliance with Good Medical Practice (GMP). The 
allocation sequence is generated by Glostrup Pharmacy using computer-generated random numbers in blocks of 10. Treatment may be unblinded in medical emergencies during the study if the investigators deem it necessary. Unblinding during the study period may be made individually and is performed by telephone contact from the investigators to Glostrup Pharmacy, where the allocation sequence is stored. A copy of the allocation sequence is concealed in opaque, sealed envelopes which are stored in a locked cabinet in the sponsor's office. Data analysis will be blinded to the investigators regarding primary, exploratory, and secondary endpoints (triple blinding).

\section{Study organization}

\section{Study centers and time schedule}

Patients are recruited from specialized HF clinics at five sites in Denmark (Herlev-Gentofte Hospital, Odense University Hospital, Bispebjerg-Frederiksberg Hospital, Rigshospitalet, and Amager-Hvidovre Hospital). Screening, randomization, and protocol-specified assessments are performed at two sites (Herlev-Gentofte Hospital and Odense University Hospital). All assessments are performed at both sites, except RHC, which is only performed in patients randomized at Odense University Hospital and DXA-scan, and ${ }^{51} \mathrm{Cr}$-EDTA clearance and dobutamine stress-echocardiography, which are only performed in patients randomized at Herlev-Gentofte Hospital (Fig. 2). The study protocol and the used methods are routine procedures at the performing sites [40, 41]. At present (December 2018), 112 patients have been randomized and enrolment follows the planned schedule. It is expected that the last patient's last visit will be in October 2019.

\section{Steering committee}

The steering committee consists of JJ, MO, CK, MKP, CT, IG, LK (chair), FG, EF, NEB, LV, JEM, and MS. The steering committee is responsible for the design, monitoring, reporting, and publication of the trial. Primary investigators are MS at Herlev-Gentofte Hospital and JEM at Odense University Hospital. The steering committee will have access to the final trial dataset.

\section{Monitoring and data collection}

Data will be collected and stored using electronic case report forms (eCRFs) constructed in the Research Electronic Data Capture (REDCap) system (Vanderbilt University (C2018). Corresponding source documents are stored at the experimental sites in accordance with the rules and regulations of the Danish Data Protection Agency to ensure confidentiality. The study is monitored by the GCP units at the University of Copenhagen and the University of Southern Denmark based on a specific monitoring plan. The GCP units are independent from the steering committee.

\section{Ethics and adverse events}

The safety of the randomized patients will be monitored continuously based on recording of AEs and severe adverse events (SAEs) from signing the informed consent form through four weeks after the end-of-study visit. The data will be collected and recorded on standardized forms at each contact. After the end-of-study visit, no planned contacts are performed but patients are instructed to contact the investigators if late-occurring AEs are suspected. These data are reported to the relevant authorities in accordance with applicable laws and International Conference of Harmonization Good Clinical Practice (ICH-GCP) guidelines. An independent endocrinologist is the unblinded data monitor and will evaluate the AEs and SAEs when half the patients are enrolled and can make the final decision to terminate the trial based on these safety data. Previously, no hypoglycemic events were observed when HF patients without T2D were included in a trial at Herlev-Gentofte Hospital evaluating the anti-glycemic drug Liraglutide [42]. Empagliflozin is approved for treatment of T2D. As the mechanisms behind the cardioprotective effects of empagliflozin are unknown, the steering committee finds it ethically acceptable to test active medication against placebo, instead of an active comparator. There are ongoing RCTs evaluating SGLT2 inhibitors in HFrEF patients both with and without T2D (Empagliflozin Outcome Trial in Patients with Chronic Heart Failure with Reduced Ejection Fraction [EMPEROR-Reduced], ClinicalTrials.gov Identifier NCT03057977; Study to Evaluate the Effect of Dapagliflozin on the Incidence of Worsening Heart Failure or Cardiovascular Death in Patients with Chronic Heart Failure [DAPA HF], ClinicalTrials.gov Identifier NCT03036124). The risk of significant side effects to empagliflozin is estimated to be modest. Compensation to those patients who suffer harm from study participation is set by the public Patient Compensation Association in Denmark.

\section{Biobank}

A research biobank is established in relation to the trial, where blood and urine samples are stored in coded form for later analysis of biomarkers. After the analyses declared in the protocol, the samples will be anonymized and the research biobank will be discontinued in accordance with the rules and regulations of the Danish Data Protection Agency. Patients are informed about the research biobank before signing the informed consent form.

\section{Dissemination of results}

The results of the study will be submitted to international peer-reviewed scientific journals, irrespective of their outcome, and the data will be made available to the public via EudraCT (www.clinicaltrialsregister.eu) and www.clinicaltrials.gov. Positive, inconclusive, and negative results will 
be presented. Furthermore, the results will be presented at scientific conferences as abstracts, oral presentations, and posters. The steering committee will assess authorship eligibility for the scientific papers related to the study based on the recommendations of the International Committee of Medical Journal Editors (ICMJE).

\section{Conclusions and clinical implications}

The anticipated results from ongoing randomized clinical trials will decide whether SGLT2 inhibitors will be a future treatment option in HFrEF patients. The Empire HF trial will complement these event-driven trials with mechanistic insight supporting clinicians and researchers in understanding the underlying mode of action of SGLT2 inhibitors including whether the observed effect on clinical outcomes is cardiac, renal, and/or metabolic, and whether SGLT2 inhibitors have an impact on patient-centered endpoints including physical activity and quality of life.

\section{Trial status}

The study is currently recruiting and enrolling participants. Protocol version 5, 5 October 2018. Start of recruitment: 29 June 2017. Approximate date when recruitment will be completed: 31 October 2019.

\section{Additional file}

Additional file 1: Checklist for the reporting of study protocols in accordance with the Standard Protocol Items: Recommendations for Interventional Trials (SPIRIT). (DOCX $50 \mathrm{~kb}$ )

\section{Abbreviations}

(e)ECV: (Estimated) extracellular volume; (e)GFR: (Estimated) glomerular filtration rate; (e)PV: (Estimated) plasma volume; ${ }^{51} \mathrm{Cr}$-EDTA: Chromium-51 labeled ethylenediamine tetraacetic acid; AE: Adverse event; ANCOVA: Analysis of covariance; CANVAS: Canagliflozin cardiovascular assessment study; Cl: Cardiac index; CRT-D: Cardiac resynchronization therapy with defibrillator; CRT-P: Cardiac resynchronization therapy without defibrillator; DAPA HF: Study to evaluate the effect of dapagliflozin on the incidence of worsening heart failure or cardiovascular death in patients with chronic heart failure; DECLARE-TIMI 58: Dapagliflozin effect on cardiovascular events-thrombolysis in myocardial infarction 58; DXA: Dual-energy X-ray absorptiometry; eCRF: Electronic case report form; EMPA-REG OUTCOME: Empagliflozin, cardiovascular outcomes, and mortality in type 2 diabetes; EMPEROR-Reduced: Empagliflozin outcome trial in patients with chronic heart failure with reduced ejection fraction; EQ-5D-5 L: EuroQol 5-dimension 5-level; GCP: Good clinical practice; GMP: Good medical practice; HbA1c: Hemoglobin A1c; HF: Heart failure; HFpEF: Heart failure with preserved ejection fraction; HFrEF: Heart failure with reduced ejection fraction; hs-cTNI: High-sensitivity cardiac troponin I; ICD: Implantable cardioverter defibrillator; ICH-GCP: International conference of harmonization good clinical practice; ICMJE: International committee of medical journal editors; IP: Investigational product; IQR: Interquartile range; ITT: Intention-to-treat; KCCQ: Kansas City cardiomyopathy questionnaire; LV: Left ventricular; LVEF: Left ventricular ejection fraction; LV-GLS: Left ventricular global longitudinal strain; MR-proADM: Mid-region pro-peptide of adrenomedullin; NT-proBNP: N-terminal pro-brain natriuretic peptide; NYHA: New York heart association; OGTT: Oral glucose tolerance test; PCWP: Pulmonary capillary wedge pressure; REDCap: Research electronic data capture; RHC: Right heart catheterization; SAE: Severe adverse event; SD: Standard deviation; SGLT2: Sodium-glucose cotransporter 2; SPIRIT: Standard Protocol Items: Recommendations for Interventional Trials; T2D: Type 2 diabetes; UACR: Urine albumin to creatinine ratio

\section{Acknowledgements}

Not applicable.

\section{Authors' contributions}

$J$ J, MO, JEM, and MS participate in study concept and design, study operations, and manuscript preparation. CK, MKP, CT, IG, LK, FG, EF, NEB, and LV participated in study concept and design, and in manuscript preparation. PHF participated in study operations. All authors read and approved the final manuscript.

\section{Authors' information}

MS is a consultant cardiologist at the Department of Cardiology, HerlevGentofte Hospital, and associate professor at the Faculty of Health and Medical Sciences, Copenhagen University. MS is the sponsor of the study. MS and JEM are the primary investigators at Herlev-Gentofte Hospital and Odense University Hospital, respectively. JJ is the contact for public queries and delegated contact for scientific queries (email address: jesper.jensen.06@regionh.dk. Telephone number: + 4538686258. Postal address: Department of Cardiology, HerlevGentofte Hospital, Herlev Ringvej 75, 2730 Herlev, DK).

\section{Funding}

Organizations funding the project include the Research Council at HerlevGentofte Hospital; FUKAP at the Department of Cardiology at HerlevGentofte Hospital; the Capital Region of Denmark (application number A6058); the Danish Heart Foundation (grant numbers 17-R116-A7714-22076 and 18-R124-A8573-22107); and the A.P. Møller Foundation for the Advancement of Medical Science (journal number 17-L-0002). None of the funding organizations are involved in the design of the study, data collection, management, or analysis of the data, and are not involved in writing the manuscript or the decision to submit the manuscript for publication.

\section{Availability of data and materials}

The datasets used and/or analyzed during the current study are available from the corresponding author on reasonable request.

\section{Ethics approval and consent to participate}

All patients give written informed consent before enrolment. The trial, including the consent form and all other related documentation given to patients, is approved by the Regional Committee on Health Research Ethics, Capital Region of Denmark (reference number H-17010756).

\section{Consent for publication}

Not applicable.

\section{Competing interests}

JJ, MO, CK, MKP, CT, IG, LK, EF, NEB, LV, PHF, and JEM declare no conflicts of interest. FG reports lecture fee from Boehringer Ingelheim. MS reports lecture fee from Novo Nordisk and Boehringer Ingelheim.

\section{Author details}

'Department of Cardiology, Herlev-Gentofte Hospital, Herlev Ringvej 75, 2730 Herlev, DK, Denmark. ${ }^{2}$ Department of Cardiology, Odense University Hospital, J. B. Winsløws Vej 4, 5000 Odense C, DK, Denmark. ${ }^{3}$ Department of Endocrinology, Rigshospitalet, Blegdamsvej 9, 2100 København $\varnothing$, DK, Denmark. ${ }^{4}$ Department of Cardiology, Bispebjerg-Frederiksberg Hospital, Nordre Fasanvej 57, 2000 Frederiksberg, DK, Denmark. ${ }^{5}$ Department of Cardiology, The Heart Centre, Rigshospitalet, Blegdamsvej 9, 2100 København $\varnothing$, DK, Denmark. ${ }^{6}$ Department of Cardiology, Zealand University Hospital, Sygehusvej 10, 4000 Roskilde, DK, Denmark. ${ }^{7}$ Faculty of Health and Medical Sciences, Copenhagen University, Blegdamsvej 3B, 2200 København N, DK, Denmark. ${ }^{8}$ Faculty of Health Sciences, University of Southern Denmark, J.B. Winsløws Vej 19, 3, 5000 Odense C, DK, Denmark. ${ }^{9}$ Clinical Institute, Aalborg University, Søndre Skovvej 15, 9000 Aalborg, DK, Denmark.

Received: 9 January 2019 Accepted: 27 May 2019

Published online: 21 June 2019

References

1. Zinman B, Wanner C, Lachin JM, Fitchett D, Bluhmki E, Hantel S, et al. Empagliflozin, cardiovascular outcomes, and mortality in type 2 diabetes. N Engl J Med. 2015;373(22):2117-28. 
2. Neal B, Perkovic V, Matthews DR. Canagliflozin and cardiovascular and renal events in type 2 diabetes. N Engl J Med. 2017;377(21):2099.

3. Wiviott SD, Raz I, Bonaca MP, Mosenzon O, Kato ET, Cahn A, et al. Dapagliflozin and cardiovascular outcomes in type 2 diabetes. N Engl J Med. 2019;380(4):347-57.

4. Kosiborod M, Cavender MA, Fu AZ, Wilding JP, Khunti K, Holl RW, et al. Lower risk of heart failure and death in patients initiated on sodium-glucose cotransporter-2 inhibitors versus other glucose-lowering drugs: The CVD-REAL Study (Comparative Effectiveness of Cardiovascular Outcomes in New Users of Sodium-Glucose Cotransporter-2 Inhibitors). Circulation. 2017;136(3):249-59.

5. Kosiborod M, Lam CSP, Kohsaka S, Kim DJ, Karasik A, Shaw J, et al. Cardiovascular events associated with SGLT-2 inhibitors versus other glucoselowering drugs: The CVD-REAL 2 Study. J Am Coll Cardiol. 2018;71 (23):2628-39.

6. Verma S, MCMurray JJV. SGLT2 inhibitors and mechanisms of cardiovascular benefit: a state-of-the-art review. Diabetologia. 2018;61(10):2108-17.

7. Zelniker TA, Braunwald E. Cardiac and renal effects of sodium-glucose cotransporter 2 inhibitors in diabetes: JACC state-of-the-art review. J Am Coll Cardiol. 2018;72(15):1845-55.

8. Lytvyn Y, Bjornstad P, Udell JA, Lovshin JA, Cherney DZI. Sodium glucose cotransporter-2 inhibition in heart failure: potential mechanisms, clinical applications, and summary of clinical trials. Circulation. 2017;136(17):1643-58.

9. Packer M, Anker SD, Butler J, Filippatos G, Zannad F. Effects of sodium-glucose cotransporter 2 inhibitors for the treatment of patients with heart failure: proposal of a novel mechanism of action. JAMA Cardiol. 2017;2(9):1025-9.

10. Fitchett D, Zinman B, Wanner C, Lachin JM, Hantel S, Salsali A, et al. Heart failure outcomes with empagliflozin in patients with type 2 diabetes at high cardiovascular risk: results of the EMPA-REG OUTCOME(R) trial. Eur Heart J. 2016;37(19):1526-34.

11. Radholm K, Figtree G, Perkovic V, Solomon SD, Mahaffey KW, de Zeeuw D, et al. Canagliflozin and Heart Failure in Type 2 Diabetes Mellitus: Results From the CANVAS Program (Canagliflozin Cardiovascular Assessment Study). Circulation. 2018;138(5):458-68.

12. Al-Jobori H, Daniele G, Cersosimo E, Triplitt C, Mehta R, Norton L, et al. Empagliflozin and kinetics of renal glucose transport in healthy individuals and individuals with type 2 diabetes. Diabetes. 2017;66(7):1999-2006.

13. Hallow KM, Helmlinger G, Greasley PJ, McMurray JJV, Boulton DW. Why do SGLT2 inhibitors reduce heart failure hospitalization? A differential volume regulation hypothesis. Diabetes Obes Metab. 2018;20(3):479-87.

14. Inzucchi SE, Zinman B, Fitchett D, Wanner C, Ferrannini E, Schumacher M, et al. How does empagliflozin reduce cardiovascular mortality? Insights from a mediation analysis of the EMPA-REG OUTCOME Trial. Diabetes Care. 2018:41(2):356-63.

15. Bettencourt P, Azevedo A, Pimenta J, Frioes F, Ferreira S, Ferreira A. $\mathrm{N}$-terminal-pro-brain natriuretic peptide predicts outcome after hospital discharge in heart failure patients. Circulation. 2004;110(15):2168-74.

16. Gardner RS, Ozalp F, Murday AJ, Robb SD, McDonagh TA. N-terminal probrain natriuretic peptide. A new gold standard in predicting mortality in patients with advanced heart failure. Eur Heart J. 2003;24(19):1735-43.

17. Schou M, Gustafsson F, Nielsen PH, Madsen LH, Kjaer A, Hildebrandt PR. Unexplained week-to-week variation in BNP and NT-proBNP is low in chronic heart failure patients during steady state. Eur J Heart Fail. 2007:9(1):68-74.

18. Ferrannini E, Baldi S, Frascerra S, Astiarraga B, Heise T, Bizzotto R, et al. Shift to fatty substrate utilization in response to sodium-glucose cotransporter 2 inhibition in subjects without diabetes and patients with type 2 diabetes. Diabetes. 2016;65(5):1190-5.

19. Ferrannini E, Mark M, Mayoux E. CV Protection in the EMPA-REG OUTCOME Trial: A "Thrifty Substrate" Hypothesis. Diabetes Care. 2016;39(7):1108-14.

20. Ponikowski P, Voors AA, Anker SD, Bueno H, Cleland JGF, Coats AJS, et al. 2016 ESC Guidelines for the diagnosis and treatment of acute and chronic heart failure: The Task Force for the diagnosis and treatment of acute and chronic heart failure of the European Society of Cardiology (ESC)Developed with the special contribution of the Heart Failure Association (HFA) of the ESC. Eur Heart J. 2016;37(27):2129-200

21. Egstrup M, Schou M, Gustafsson I, Kistorp CN, Hildebrandt PR, Tuxen CD. Oral glucose tolerance testing in an outpatient heart failure clinic reveals a high proportion of undiagnosed diabetic patients with an adverse prognosis. Eur J Heart Fail. 2011;13(3):319-26.

22. Kistorp C, Galatius S, Gustafsson F, Faber J, Corell P, Hildebrandt P. Prevalence and characteristics of diabetic patients in a chronic heart failure population. Int J Cardiol. 2005;100(2):281-7.

23. Redfield MM, Anstrom KJ, Levine JA, Koepp GA, Borlaug BA, Chen HH, et al. Isosorbide Mononitrate in Heart Failure with Preserved Ejection Fraction. N Engl J Med. 2015;373(24):2314-24.
24. Christensen HM, Kistorp C, Schou M, Keller N, Zerahn B, Frystyk J, et al. Prevalence of cachexia in chronic heart failure and characteristics of body composition and metabolic status. Endocrine. 2013;43(3):626-34.

25. Duarte K, Monnez JM, Albuisson E, Pitt B, Zannad F, Rossignol P. Prognostic value of estimated plasma volume in heart failure. JACC Heart Fail. 2015; 3(11):886-93.

26. Fritzsche I, Buhrdel P, Melcher R, Bohme HJ. Stability of ketone bodies in serum in dependence on storage time and storage temperature. Clin Lab. 2001;47(7-8):399-403.

27. Laun RA, Rapsch B, Abel W, Schroder O, Roher HD, Ekkernkamp A, et al. The determination of ketone bodies: preanalytical, analytical and physiological considerations. Clin Exp Med. 2001;1(4):201-9.

28. Smith GL, Lichtman JH, Bracken MB, Shlipak MG, Phillips CO, DiCapua P, et al. Renal impairment and outcomes in heart failure: systematic review and meta-analysis. J Am Coll Cardiol. 2006;47(10):1987-96.

29. Brøchner-Mortensen J. A simple single injection method for determination of the extracellular fluid volume. Scand J Clin Lab Invest. 1980;40(6):567-73.

30. Jackson CE, Solomon SD, Gerstein HC, Zetterstrand S, Olofsson B, Michelson $\mathrm{EL}$, et al. Albuminuria in chronic heart failure: prevalence and prognostic importance. Lancet. 2009;374(9689):543-50.

31. Anker SD, Doehner W, Rauchhaus M, Sharma R, Francis D, Knosalla C, et al. Uric acid and survival in chronic heart failure: validation and application in metabolic, functional, and hemodynamic staging. Circulation. 2003;107(15):1991-7.

32. Tsutamoto T, Kawahara C, Nishiyama K, Yamaji M, Fujii M, Yamamoto T, et al. Prognostic role of highly sensitive cardiac troponin I in patients with systolic heart failure. Am Heart J. 2010:159(1):63-7.

33. von Haehling S, Filippatos GS, Papassotiriou J, Cicoira M, Jankowska EA, Doehner W, et al. Mid-regional pro-adrenomedullin as a novel predictor of mortality in patients with chronic heart failure. Eur J Heart Fail. 2010;12(5):484-91.

34. Paraskevaidis IA, Ikonomidis I, Simitsis P, Parissis J, Stasinos V, Makavos G, et al. Multidimensional contractile reserve predicts adverse outcome in patients with severe systolic heart failure: a 4-year follow-up study. Eur J Heart Fail. 2017:19(7):846-61.

35. Motoki H, Borowski AG, Shrestha K, Troughton RW, Tang WH, Thomas JD, et al. Incremental prognostic value of assessing left ventricular myocardial mechanics in patients with chronic systolic heart failure. J Am Coll Cardiol. 2012:60(20):2074-81.

36. Patel CB, DeVore AD, Felker GM, Wojdyla DM, Hernandez AF, Milano CA, et al. Characteristics and outcomes of patients with heart failure and discordant findings by right-sided heart catheterization and cardiopulmonary exercise testing. Am J Cardiol. 2014;114(7):1059-64.

37. Heidenreich PA, Spertus JA, Jones PG, Weintraub WS, Rumsfeld JS, Rathore SS, et al. Health status identifies heart failure outpatients at risk for hospitalization or death. J Am Coll Cardiol. 2006:47(4):752-6.

38. Mitchell PM, Al-Janabi H, Richardson J, lezzi A, Coast J. The relative impacts of disease on health status and capability wellbeing: a multi-country study. PLoS One. 2015;10(12):e0143590.

39. Andersen MJ, Ersboll M, Bro-Jeppesen J, Moller JE, Hassager C, Kober L, et al. Relationships between biomarkers and left ventricular filling pressures at rest and during exercise in patients after myocardial infarction. J Card Fail. 2014;20(12):959-67.

40. Andersen MJ, Ersboll M, Axelsson A, Gustafsson F, Hassager C, Kober L, et al. Sildenafil and diastolic dysfunction after acute myocardial infarction in patients with preserved ejection fraction: the Sildenafil and Diastolic Dysfunction After Acute Myocardial Infarction (SIDAMI) trial. Circulation. 2013;127(11):1200-8

41. Jorsal A, Wiggers H, Holmager P, Nilsson B, Nielsen R, Boesgaard TW, et al. A protocol for a randomised, double-blind, placebo-controlled study of the effect of Llraglutide on left VEntricular function in chronic heart failure patients with and without type 2 diabetes (The LIVE Study). BMJ Open. 2014;4(5):e004885.

42. Jorsal A, Kistorp C, Holmager P, Tougaard RS, Nielsen R, Hanselmann A, et al. Effect of liraglutide, a glucagon-like peptide-1 analogue, on left ventricular function in stable chronic heart failure patients with and without diabetes (LIVE)-a multicentre, double-blind, randomised, placebo-controlled trial. Eur J Heart Fail. 2017;19(1):69-77.

\section{Publisher's Note}

Springer Nature remains neutral with regard to jurisdictional claims in published maps and institutional affiliations. 